\title{
Observing hydrogen silsesquioxane cross-linking with broadband CARS
}

\author{
Allison G. Caster, ${ }^{a}$ Stefan Kowarik, ${ }^{a}$ Adam M. Schwartzberg, ${ }^{a}$ \\ Olivier Nicolet, ${ }^{\text {b }}$ Sang-Hyun Lim ${ }^{\text {c }}$ and Stephen R. Leone ${ }^{\text {a* }}$
}

\begin{abstract}
Broadband coherent anti-Stokes Raman scattering microscopy (CARS microscopy) is used for real-time measurements of in situ cross-linking of the photoresist material hydrogen silsesquioxane (HSQ). Using samples baked at different temperatures, and therefore cross-linked to varying degrees, characteristic Raman bands are identified for the open 'cage' structure (345 $\mathrm{cm}^{-1}$, $454 \mathrm{~cm}^{-1}$ and $\left.562 \mathrm{~cm}^{-1}\right)$ as well as the cross-linked 'network' structure $\left(484 \mathrm{~cm}^{-1}\right)$. Cross-linking can also be induced by absorption of high intensity near-infrared (IR) laser light $\left(\sim 10^{13} \mathrm{~W} \mathrm{~cm}^{-2}\right)$ and followed in real time by monitoring the characteristic Raman bands. Using the time resolution of CARS to follow the HSQ reaction during the laser exposure, a two-stage kinetic process is observed and associated time constants of $20 \pm 10 \mathrm{~s}$ and $3 \pm 1$ min are determined for the IR laser-induced cross-linking in films of $\sim 1 \mu \mathrm{m}$ thickness. Copyright (c) 2009 John Wiley \& Sons, Ltd.
\end{abstract}

Keywords: coherent anti-Stokes Raman scattering; CARS; hydrogen silsesquioxane; POSS; photolithography

\section{Introduction}

The ability to make increasingly smaller and thinner interlayer dielectrics (ILD) is an important limiting factor in the quest for more compact electronics. New photoresist materials, such as hydrogen silsesquioxane (HSQ), can be suspended in a solution with a volatile solvent and deposited by spin-coating the solution onto the substrate. These 'spin-on dielectrics' are very promising for simplifying the methods and time needed to produce smaller electronics and thereby also reducing the cost. ${ }^{[1-4]} \mathrm{HSQ}$ as a monomer has a nominal cage structure with a $\mathrm{Si}-\mathrm{H}$ at each corner, bridged by oxygen [Fig. 1(a)]. Upon e-beam, ${ }^{[4,5]}$ ultra-violet or $X$-ray exposure, ${ }^{[6]}$ or heating, ${ }^{[7-10]}$ the monomer cages partially cross-link into a network structure by forming additional bonds between cages, thus becoming more glass like [Fig. 1(b)]. This cross-linking may involve reactions with water vapor to produce $\mathrm{OH}$ groups on the $\mathrm{Si}-\mathrm{H}$ corners, which subsequently react to form $\mathrm{Si}-\mathrm{O}-\mathrm{Si}$ bridges. ${ }^{[4,5]}$ Nanoscale features can be patterned in the film, which have a different solubility than the surrounding parent material, and traditional wet lithography can be used to remove the unexposed regions.

However, there are challenges yet to be surmounted in using HSQ and its derivatives commercially as photoresists, such as irreproducibility and reaction front propagation beyond the exposed region. ${ }^{[1,5]}$ There have been a variety of studies that aim to determine the mechanism by which the network structure forms ${ }^{[4,7,10,11]}$ but there are still gaps in understanding, and there are only very limited means for monitoring the cross-linking process as it occurs. In order to solve some of the problems for producing desired features in HSQ for nanopatterning, ILD, and other applications, it is necessary to understand the fundamental chemistry taking place during the cross-linking. This is where broadband coherent anti-Stokes Raman scattering (CARS) can play a key role.

In the CARS process, two fields, called the pump and Stokes fields, interact with the sample to induce coherent molecular vibrations. A third probe field then scatters off of these vibrations to produce the coherent anti-Stokes Raman signal. While very sensitive and rapid measurements can be made with CARS microscopy using multiple beams, ${ }^{[12-16]}$ the essential feature of the broadband method employed here is the use of a single, ultra-broadband pulsed laser, which simultaneously provides all three photons necessary to produce the CARS signal. ${ }^{[17-20]}$ Because the entire signal is obtained from just a single laser beam, there are no complications due to frequency tuning, timing jitter or alignment between multiple beams. Broadband CARS microscopy can enable rapid analysis of multiple Raman bands, especially when compared to spontaneous Raman spectroscopy, while providing the full fingerprint region Raman-equivalent spectrum. ${ }^{[21-23]}$ The faster spectral acquisition allows the possibility to monitor changes to the Raman spectrum as the HSQ film undergoes its beam-induced transformation from monomer cages to the cross-linked (network) structure, the process utilized for patterning the films. To our knowledge, the time scale of the fastest kinetics studies on HSQ cross-linking to date are in minutes, compiled by taking Fourier transform infrared (FTIR) spectra of HSQ films baked for varied amounts of time at different temperatures. ${ }^{[9,10]}$ Although most of the cross-linking occurs within the first minutes of heating, the time resolution of these measurements is limited by the sample heating and cooling times. However, with broadband CARS, spectra can be acquired in situ on a sub-second time scale.

Correspondence to: Stephen R. Leone, Department of Chemistry and Physics,
University of California, and Lawrence Berkeley National Laboratory, Berkeley, CA 94720, USA.E-mail:srl@berkeley.edu

a Department of Chemistry and Physics, University of California, and Lawrence Berkeley National Laboratory, Berkeley, CA 94720, USA

b Nestlé Product Technology Center, 1350 Orbe, Switzerland

c Department of Chemistry and Biochemistry, University of Texas, Austin, TX 78712, USA 
(a)

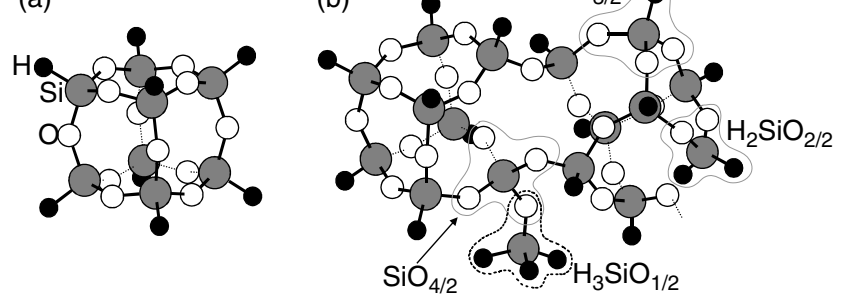

Figure 1. Structure of HSQ (a) monomer (cage) form and (b) partially crosslinked (network) form, showing some intermediate species.

In this article we show that cross-linking of HSQ can be induced by the high intensity of a focused near-infrared (IR) laser beam and monitored in real time by following the changes in the Raman peaks that correspond to the cage and network structures. By taking a spectrum every $500 \mathrm{~ms}$, we are able to observe in greater detail the initial fast kinetics, which demonstrates a two-stage cross-linking mechanism.

\section{Experimental}

Hydrogen silsesquioxane $\left[\mathrm{H}_{8} \mathrm{Si}_{8} \mathrm{O}_{12}\right.$, also indicated as polyhedral oligomeric silsesquioxane (POSS)] films were prepared from a flowable oxide solution (FOx-25, DOW Corning, 15-40 wt\% HSQ in a volatile methyl siloxane solvent). Thin films were produced by spin-coating $120 \mu \mathrm{l}$ of the solution onto glass microscope coverslips at $800 \mathrm{rpm}$ for $30 \mathrm{~s}$, yielding films of approximately $1-1.2 \mu \mathrm{m}$ in thickness, as measured with profilometry. Films were then cured on a hot plate in air for $10 \mathrm{~min}$ at $150^{\circ} \mathrm{C}$ to evolve any remaining solvent. All samples were stored under high nitrogen flow when not being measured. For temperaturedependent measurements, samples were baked for $10 \mathrm{~min}$ at $150^{\circ} \mathrm{C}, 250^{\circ} \mathrm{C}, 300^{\circ} \mathrm{C}, 350^{\circ} \mathrm{C}$ and $450^{\circ} \mathrm{C} \pm 15^{\circ} \mathrm{C}$.

To obtain coherent Raman spectra of the thin film samples, we used the broadband CARS setup shown in Fig. 2. All three electric fields for the CARS process are generated in a single pulse by a Ti:Sapphire laser oscillator (KM Labs) operating at $90 \mathrm{MHz}$, providing approximately 20 -fs pulses with $120 \mathrm{~nm}$ of bandwidth centered at $800 \mathrm{~nm}$. After a telescope, the beam passes through an all-reflective $4 \mathrm{f}$ geometry pulse shaper. Within the pulse shaper, the light is first dispersed into the Fourier plane by a $600-$ grooves $/ \mathrm{mm}$ diffraction grating, then collimated and passed through a dualmask 640 pixel spatial light modulator (SLM-640-D, CRI), which is configured for phase and polarization shaping. Most of the broad spectrum passes through unchanged, and these spectral components act as the pump and Stokes fields for the CARS process. A narrow probe field is differentiated within the beam by applying a $90^{\circ}$ phase and polarization change to a narrow spectral region with the SLM. For these experiments, a probe centered at $759 \mathrm{~nm}$ with a width of $\sim 1 \mathrm{~nm}$ was employed, corresponding to a spectral resolution of about $17 \mathrm{~cm}^{-1}$.

After the SLM, the beam is again collimated, and a second grating compresses it back into a short pulse. The light is then focused onto the sample through a 1.2 NA water-immersion objective (60× UPlanApo, Olympus), and the signal is collected through a 0.85 NA air objective or 1.0 NA water objective. The average beam power is $46 \mathrm{~mW}$ before the microscope, focused to a diffraction limited spot of $\sim 400 \mathrm{~nm}$ diameter, giving an approximate peak intensity of $2 \times 10^{13} \mathrm{~W} \mathrm{~cm}^{-2}$. The laser light is removed by a

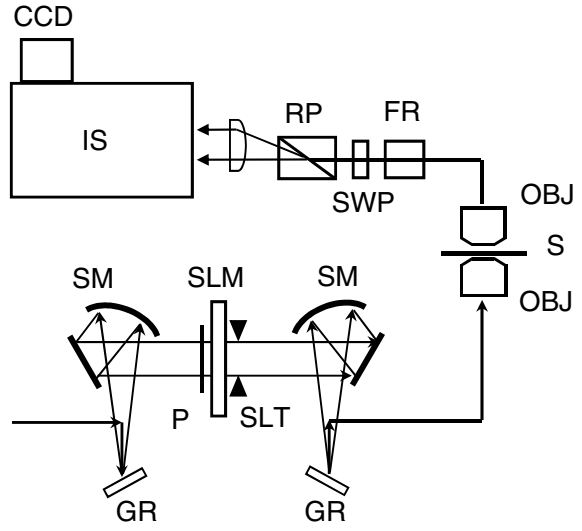

Figure 2. Experimental setup for broadband DQSI CARS. GR, grating; $S M$, spherical mirror; P, polarizer; SLM, spatial light modulator; SLT, slit; OBJ, microscope objective; $S$, sample; FR, Fresnel rhomb; SWP, short-wave pass filter; RP, Rochon prism; IS, imaging spectrometer; CCD, two-dimensional charge-coupled device.

sharp-edge short-wave pass filter (740 AESP, Omega Optical), and the overall polarization is rotated by $45^{\circ}$ by an achromatic halfwave plate (Fresnel Rhomb, CVI). A Rochon prism (CVI) splits the signal into two traces of horizontal and vertical polarization, which are focused into a spectrometer (SP2300i, PI-Acton) and imaged simultaneously by a two-dimensional CCD detector (DV401-FI, Andor).

The total CARS signal is given by

$$
S \propto\left|P_{R}+P_{N R}\right|^{2}=P_{R}^{*} P_{R}+P_{N R}^{2}+P_{N R}\left(P_{R}^{*}+P_{R}\right)
$$

where $P_{\mathrm{R}}$ and $P_{\mathrm{NR}}$ are the resonant and nonresonant transient polarizations, respectively. Due to the broad bandwidth of the laser, the resonant Raman signal is accompanied by a large nonresonant background, $P_{\mathrm{NR}}$, which is several orders of magnitude larger than the desired resonant signal, induced by four-wave mixing within the sample itself. ${ }^{[13]}$ However, the resonant and nonresonant signals are coherent. To detect the weak Raman signal, it is therefore necessary to either suppress the nonresonant background or use the nonresonant light for homodyne amplification of the resonant signal. ${ }^{[18-20]}$ By acquiring two projections of the signal simultaneously, it is possible to measure both the nonresonant background and the homodyne-amplified resonant CARS signal (the cross-term in the above equation) using double-quadrature spectral interferometry (DQSI). ${ }^{[24]}$ The resonant CARS signal (i.e. the imaginary part of the cross-term in the equation above) is obtained by taking the normalized difference

$$
D(\omega)=\frac{S^{i}(\omega)-S^{j}(\omega)}{\sqrt{S^{i}(\omega)+S^{j}(\omega)}}
$$

where $S^{i}$ and $S^{j}$ are the top and bottom projections of the signal on the CCD, yielding the resonant signal $D(\omega)$ in units of $\sqrt{\text { counts. }}{ }^{[19]}$

Significant spectral phase (chirp) is introduced by the focusing objective, which must be compressed at the focus to ensure the short duration and broad bandwidth of the laser pulse at the sample position. Prior to taking spectra, the first and second order components of the chirp are compressed with the appropriate phase mask on the SLM by optimizing the intensity and bandwidth of the total nonresonant signal from the sample itself. In this study, 
homodyne-amplified CARS spectra were collected, and the region from 300 to $675 \mathrm{~cm}^{-1}$ was analyzed to monitor the changing HSQ bands during cross-linking. A spectral width of up to $1100 \mathrm{~cm}^{-1}$ can be achieved, as has been previously demonstrated. ${ }^{[20]}$

\section{Results and Discussion}

Broadband CARS is used to study the temperature dependency of cross-linking in HSQ and the real-time kinetics during laserinduced cross-linking. Figure 3(a) shows CARS spectra for HSQ films baked for $10 \mathrm{~min}$ at temperatures of $150^{\circ} \mathrm{C}, 250^{\circ} \mathrm{C}, 300^{\circ} \mathrm{C}$, and $450^{\circ} \mathrm{C}$. Clear spectral changes are visible with increasing baking temperature, demonstrating the chemical sensitivity of CARS with respect to the HSQ microstructure. The film baked at $150^{\circ} \mathrm{C}$ corresponds primarily to the nominal cage structure, as established in previous studies of thermal curing of HSQ, which show that the HSQ cages cross-link to the network structure only upon heating above $190^{\circ} \mathrm{C} \cdot{ }^{[7,11]}$ With increasing baking temperature, and therefore increased cross-linking, Raman bands at $342 \mathrm{~cm}^{-1}, 454 \mathrm{~cm}^{-1}$, and $562 \mathrm{~cm}^{-1}$ disappear, whereas a band characteristic of the network structure grows in at $484 \mathrm{~cm}^{-1}$. In order to identify the Raman bands in the cage and cross-linked $\mathrm{HSQ}$, we also performed spontaneous Raman spectroscopy on films baked at $150^{\circ} \mathrm{C}$ as well as $350^{\circ} \mathrm{C}$ for $10 \mathrm{~min}$. Comparison of the CARS spectra in Fig. 3(a) to spontaneous Raman spectra (not shown) demonstrates very good agreement of peak positions and lineshapes, showing that the homodyne-amplified CARS spectra are equivalent to the spontaneous Raman spectra from 300 to $675 \mathrm{~cm}^{-1}$. Further, the comparison demonstrates the higher sensitivity of the broadband CARS, which requires integration times of only $1 \mathrm{~s}$ for spectra in Fig. 3(a), as opposed to the minute timescale for spontaneous Raman spectra.

In a second set of experiments, we have utilized the time resolution possible in the CARS measurements to study the kinetics of near-IR cross-linking, as shown in Fig. 3(b). In contrast to curing the films at different temperatures for Fig. 3(a), the changes in Fig. 3(b) are induced by focusing the near-IR beam into a diffraction limited volume, with an approximate peak intensity of $2 \times 10^{13} \mathrm{~W} \mathrm{~cm}^{-2}$. While $50 \mathrm{~ms}$ integration time is sufficient for reasonable signal-to-noise ratios, the spectra as shown in Fig. 3(b) have an acquisition time of $0.5 \mathrm{~s}$, which is still fast compared to the timescales of cross-linking. With increasing near-IR exposure time, we see spectral changes similar to the ones observed upon baking the HSQ films, demonstrating that HSQ cross-linking occurs.

The clear isosbestic point at $535 \mathrm{~cm}^{-1}$ (Fig. 3) suggests the reduction of one species and the growth of another. By comparing the spectra in Fig. 3(a) and (b), one sees that the amount of conversion to the network structure after $10 \mathrm{~min}$ of near-IR exposure is greater than for the sample heated to $300{ }^{\circ} \mathrm{C}$ for $10 \mathrm{~min}$. Since it has been reported that HSQ does not have any direct sensitivity to cross-linking under near-IR exposure, ${ }^{[6]}$ we consider multiphoton absorption and heating in the focus of the intense beam as two possible conversion mechanisms. At the powers used, some heating of the sample is expected in the beam focus, but note that a temperature of at least $190^{\circ} \mathrm{C}$ must be reached to initiate bond rearrangement in HSQ. ${ }^{[1]}$ To determine whether heating was the primary mechanism, we measured the cross-linking rate using a water-immersion collection objective. If heating played a significant role in the cross-linking, we reason that the water should slow the cross-linking by better thermal removal of the heat, thus resulting in a lower temperature within
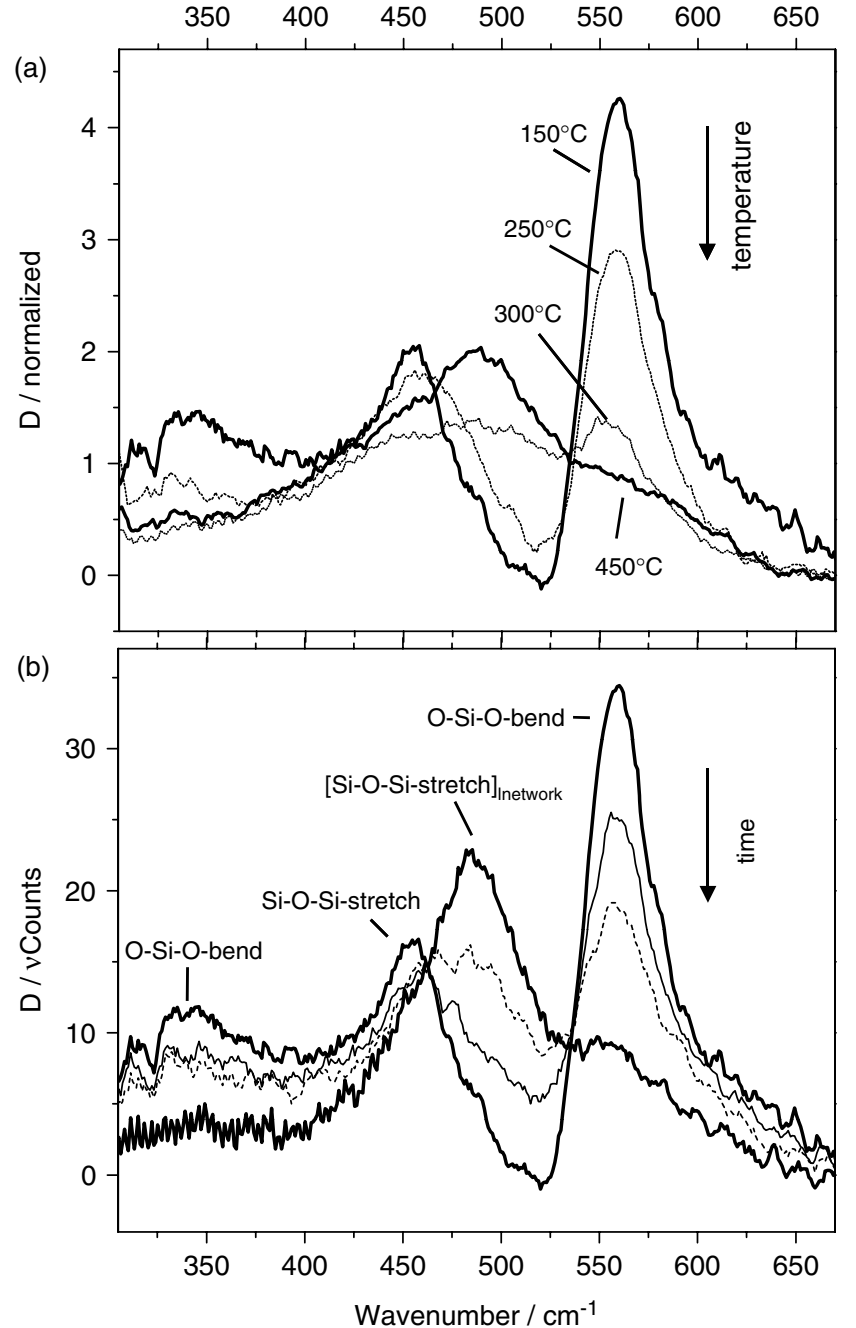

Figure 3. Coherent anti-Stokes Raman spectra of HSQ. (a) Temperature dependence of cross-linking in samples baked for $10 \mathrm{~min}$ at $150^{\circ} \mathrm{C}, 250^{\circ} \mathrm{C}$, $300^{\circ} \mathrm{C}$ or $450^{\circ} \mathrm{C}$. The HSQ cross-linking proceeds more completely at higher temperatures. (b) Real-time and in situ monitoring of cross-linking. The sample was baked for $10 \mathrm{~min}$ at $150^{\circ} \mathrm{C}$, then continuously exposed to the focused near-IR beam for the next $10 \mathrm{~min}$. Spectra were taken after 0,16 and $32 \mathrm{~s}$ and 10 min of near-IR exposure with an integration time of $500 \mathrm{~ms}$.

the focal volume. However, it was observed that when water was in contact with the sample during exposure, the cross-linking rate did not change. Furthermore, it was observed that when the average laser power was reduced by half, the rate for the initial stage of cross-linking decreased by a factor of ten. This implies a strongly nonlinear process, suggesting that the primary crosslinking mechanism in the laser focus is multiphoton photo-induced cross-linking.

Based on observations and calculations reported by Bornhauser and Calzaferri, ${ }^{[25]}$ we can assign the Raman bands in the CARS spectra of noncross-linked (cage) HSQ. However, previous Raman assignments for cross-linked (network) HSQ are not available. For the noncross-linked HSQ films, we assigned the $342 \mathrm{~cm}^{-1}$ and $562 \mathrm{~cm}^{-1}$ peaks to $\mathrm{O}-\mathrm{Si}-\mathrm{O}$ bends, compared to spontaneous Raman spectroscopy values of $341 \mathrm{~cm}^{-1}$ and $576 \mathrm{~cm}^{-1}$ (calculated). ${ }^{[25]}$ The $454 \mathrm{~cm}^{-1}$ peak was assigned to the $\mathrm{Si}-\mathrm{O}-\mathrm{Si}$ symmetric stretch, compared to $446 \mathrm{~cm}^{-1}$ (calc.). ${ }^{[25]}$ 
After 10 min of near-IR exposure, the O-Si-O bend peaks at $342 \mathrm{~cm}^{-1}$ and $562 \mathrm{~cm}^{-1}$ decrease to about $25 \%$ of their original intensities, as does the $\mathrm{Si}-\mathrm{O}-\mathrm{Si}$ symmetric stretch at $454 \mathrm{~cm}^{-1}$, while a new peak at $484 \mathrm{~cm}^{-1}$ emerges. As the HSQ cages cross-link, the structure becomes more and more like that of $\mathrm{SiO}_{2}\left(\mathrm{SiO}_{4 / 2}\right)$, as $\mathrm{Si}-\mathrm{O}-\mathrm{Si}$ bonds replace $\mathrm{Si}-\mathrm{H}$ bonds. ${ }^{[2]}$ We attribute the loss of the $\mathrm{O}-\mathrm{Si}-\mathrm{O}$ bending peaks to the change of the cage $\mathrm{O}-\mathrm{Si}-\mathrm{O}$ bonds, with an angle of approximately $109.5^{\circ},{ }^{[25]}$ to the network $\mathrm{O}-\mathrm{Si}-\mathrm{O}$ bonds, which are possibly more linear and rigid compared to the cage bonds. We tentatively assign the $484 \mathrm{~cm}^{-1}$ peak to a Si-O-Si symmetric stretch after cross-linking, as FTIR spectra show that the $\mathrm{Si}-\mathrm{O}$ stretch grows in as the network structure forms ${ }^{[3]}$ and there is a known IR-active Si-O-Si symmetric stretch for cage HSQ at $481 \mathrm{~cm}^{-1}$. ${ }^{[25]}$ We suggest that the $481 \mathrm{~cm}^{-1}$ band may become Raman active in the network structure due to changes in symmetry. Furthermore, we expect that the increased rigidity of $\mathrm{Si}-\mathrm{O}-\mathrm{Si}$ bonds in the network structure leads to an increase of the stretching wavenumber from $454 \mathrm{~cm}^{-1}$. This increasing rigidity can be pictured using a physical model of the monomer cage, which is free to stretch and compress in almost any direction, whereas the cross-linked cages become more fixed in their positions as they form more bonds to other cages and the existing network. Also, the $484 \mathrm{~cm}^{-1}$ peak is substantially more broad (FWHM $61 \mathrm{~cm}^{-1}$ ) than the initial $454 \mathrm{~cm}^{-1}$ peak (FWHM $30 \mathrm{~cm}^{-1}$ ), which may be due to the fact that network O-Si-O bonds have a variety of possible angles and bond lengths at different cross-linked sites, ${ }^{[2]}$ leading to inhomogeneous broadening of the linewidth.

For a more detailed analysis of the kinetics of cross-linking we fitted the spectra in Fig. 3(b) to determine the area of the $454 \mathrm{~cm}^{-1}, 484 \mathrm{~cm}^{-1}$, and $562 \mathrm{~cm}^{-1}$ Raman bands. Figure 4 shows the decay of the $562 \mathrm{~cm}^{-1}$ peak (O-Si-O bend) characteristic of the cage structure, and the rise of the peak at $484 \mathrm{~cm}^{-1}$ (Si-O-Si symmetric stretch) characteristic of the network structure, from 0 to $10 \mathrm{~min}$. The data is best fit by a double exponential, with a fast initial time constant of $24 \mathrm{~s}$ for the decay of the O-Si-O bend and the increase of the network Si-O-Si stretch, demonstrating a direct conversion from cage to network structure without long-lasting intermediate species. The second stage of the HSQ cross-linking kinetics occurs on a much slower timescale for which the fit gives different time constants of $220 \mathrm{~s}$ for the decay and $120 \mathrm{~s}$ for the growth, with the difference probably due to fit uncertainties and neglecting inhomogeneous broadening. We reproduced this data set on different days with several film samples, and obtained time constants for the fast regime that range between 10 and $30 \mathrm{~s}$. The variability in the experiment is most likely due to variations in the film thickness and laser peak intensity (due to incomplete chirp compression). Note that the cross-linking is slower at the rim of the Gaussian beam profile, where the exposure is lower; this effect does contribute to the shape of the kinetics measurement, but it does not change the conclusion that HSQ exhibits intrinsic twostage kinetics. This two-stage cross-linking kinetics of HSQ has also been observed in multiple experiments in our laboratories by using $X$-ray induced cross-linking and $X$-ray spectroscopic detection. The two-stage mechanisms of the cross-linking will be considered in detail in a future report on the X-ray measurements.

Possible mechanisms have been proposed for thermally induced cross-linking of $\mathrm{HSQ}\left[\mathrm{HSiO}_{3} / 2\right.$, Fig. 1(a)] above the onset of curing at $\sim 190{ }^{\circ} \mathrm{C} .{ }^{[7,8,11]}$ Redistribution of the network leading ultimately to $\mathrm{SiO}_{4 / 2}$ bond formation is the primary mechanism proposed for curing from $\sim 250$ to $350^{\circ} \mathrm{C}$.

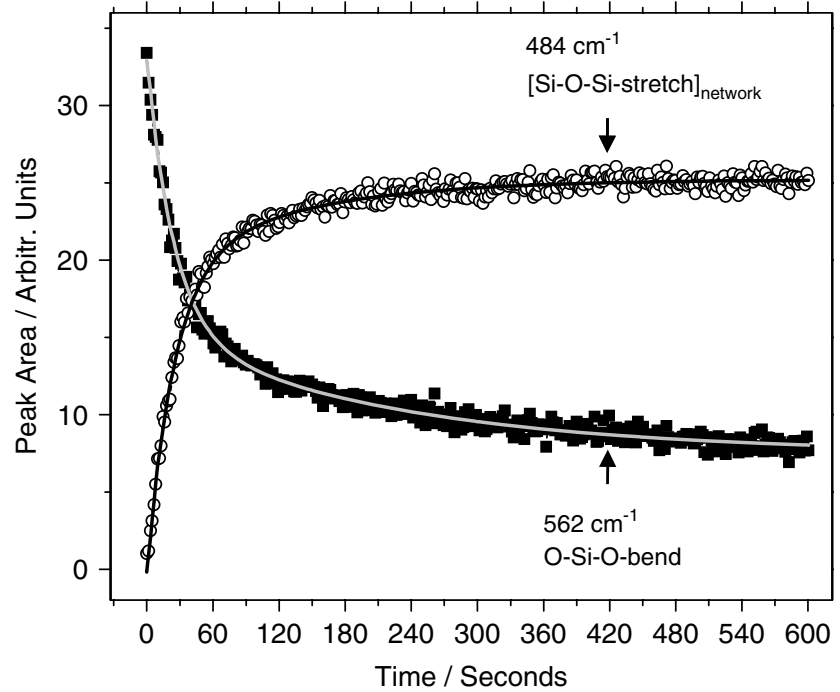

Figure 4. Decay of the $562 \mathrm{~cm}^{-1}$ peak area (O-Si-O bend) with time in the near-IR beam, and growth of the $484 \mathrm{~cm}^{-1}$ band ( $\mathrm{Si}-\mathrm{O}-\mathrm{Si}$ symmetric stretch). Solid lines are a fit to a double exponential. Both the decay and growth kinetics exhibit a fast first stage with a time constant of $24 \mathrm{~s}$ and a much slower second stage.

Remaining $\mathrm{Si}-\mathrm{H}$ bonds within the network structure [unreacted $\mathrm{HSiO}_{3 / 2}$ and intermediate species $\mathrm{H}_{2} \mathrm{SiO}_{2 / 2}$ and $\mathrm{H}_{3} \mathrm{SiO}_{1 / 2}$, Fig. 1(b)] create the porosity that gives the network HSQ its low dielectric constant. $\mathrm{SiH}_{4}$ and $\mathrm{H}_{2}$ are evolved throughout the cross-linking reaction, and the role of water vapor in converting $\mathrm{Si}-\mathrm{H}$ to $\mathrm{Si}-\mathrm{OH}$ also requires more analysis. From 350 to $435^{\circ} \mathrm{C}$, thermally induced bond dissociation, especially $\mathrm{Si}-\mathrm{H}$ bond cleavage, increases the reaction rate. Above $435^{\circ} \mathrm{C}$, the $\mathrm{Si}-\mathrm{H}$ dissociation rate increases rapidly, breaking the remaining cage-like bonds, leading to the collapse of the porous network structure. Previously it has been found that about $400^{\circ} \mathrm{C}$ is the optimal temperature for thermal curing of HSQ for use as an ILD for integrated circuits, giving the best balance of mechanical stability and low dielectric constant. ${ }^{[26]}$ First-order reaction kinetics ${ }^{[2]}$ and two-stage zero-order kinetics ${ }^{[10]}$ have both been proposed for the low temperature reaction $\left(250-350^{\circ} \mathrm{C}\right)$. The double exponential observed during near-IR exposure in this study suggests a two-stage first-order mechanism for the cross-linking. Note that the cross-linking is slower at the rim of the gaussian beam profile, where the exposure is lower; this effect does contribute to the shape of the kinetics measurement, but it does not change the conclusion that HSQ exhibits intrinsic two-stage kinetics.

\section{Conclusions}

With broadband CARS microscopy, we are able to measure crosslinking kinetics in a novel spin-on dielectric material, $\mathrm{HSQ}$, in situ in $\mathbf{5 0 0 ~ m s ~ t i m e ~ i n t e r v a l s . ~ T h e ~ r o b u s t , ~ s i n g l e - b e a m ~ i n t e r f e r o m e t r i c ~}$ method used here to acquire broad Raman-equivalent spectra allows for fast measurements that contain a great deal of spectral information.

These studies show that HSQ cross-linking occurs rapidly within the first minutes of laser-induced cross-linking with apparently first-order kinetics, followed by a very long second stage. In connection with X-ray microscopy experiments currently under way, we hope to deduce whether the reaction always follows 
two-stage first-order kinetics and to determine what species are involved in the two stages of the reaction. Improvements in spectral width and sensitivity over the past year have opened new possibilities for systems that can be studied with high spectral and spatial resolution while acquiring full Raman spectra. ${ }^{[27]}$ As we explore the possibilities of this new single-beam, broadband CARS technique, we will be able to gain more information about not only HSQ but also the properties of other rapidly changing chemical systems.

\section{Acknowledgements}

We want to thank James Schuck for experimental help with spontaneous Raman measurements, Deirdre Olynick for discussions on HSQ, and Farhad Salmassi for film thickness measurements. S. Kowarik wants to thank the Alexander von Humboldt Foundation for funding. This study was supported by the Director, Office of Science, Office of Basic Energy Sciences, of the US Department of Energy under Contract No. DE-AC02-05CH11231.

\section{References}

[1] N. Clark, A. Vanderslice, R. Grove, R. R. Krchnavek, J. Vac. Sci. Technol., B 2006, 24, 3073

[2] M. J. Loboda, C. M. Grove, R. F. Schneider, J. Electrochem. Soc. 1998 145, 2861.

[3] M. J. Loboda, G. A. Toskey, Solid State Technol. 1998, 41, 99.

[4] H. Namatsu, Y. Takahashi, K. Yamazaki, T. Yamaguchi, M. Nagase, K. Kurihara, J. Vac. Sci. Technol., B 1998, 16, 69.
[5] D. L. Olynick， J. A. Liddle， A. V. Tivanski， M. K. Gilles, T. Tyliszczak, F. Salmassi, K. Liang, S. R. Leone, J. Vac. Sci. Technol., B 2006, 24, 3048.

[6] M.Peuker, M. H.Lim, H.I.Smith, R. Morton, A. K. van LangenSuurling, J. Romijn, E. van der Drift, F. van Delft, Microelectron. Eng. 2002, 61-2, 803 .

[7] M. G. Albrecht, C. Blanchette, J. Electrochem. Soc. 1998, 145, 4019.

[8] V. Belot, R. Corriu, D. Leclercq, P. H. Mutin, A. Vioux, Chem. Mater. $1991,3,127$.

[9] W. C. Liu, C. C. Yang, W. C. Chen, B. T. Dai, M. S. Tsai, J. Non-Cryst. Solids 2002, 311, 233.

[10] C. C. Yang, W. C. Chen, J. Mater. Chem. 2002, 12, 1138

[11] Y. K. Siew, G. Sarkar, X. Hu, J. Hui, A. See, C. T. Chua, J. Electrochem. Soc. 2000, 147, 335

[12] A. Zumbusch, G. R. Holtom, X. S. Xie, Phys. Rev. Lett. 1999, 82, 4142.

[13] J. H. Wang, W. J. Chen, T. C. Chang, P. T. Liu, S. L. Cheng, J. Y. Lin, L. J. Chen, J. Electrochem. Soc. 2003, 150, F141.

[14] C. L. Evans, E. O. Potma, M. Puoris'haag, D. Cote, C. P. Lin, X. S. Xie, Proc. Natl. Acad. Sci. U.S.A. 2005, 102, 16807.

[15] M. Müller, A. Zumbusch, ChemPhysChem 2007, 8, 2157.

[16] A. Volkmer, J. Phys. D: Appl. Phys. 2005, 38, R59.

[17] N. Dudovich, D. Oron, Y. Silberberg, Nature 2002, 418, 512.

[18] A. G. Caster, S. H. Lim, O. Nicolet, S. R. Leone, Spectroscopy 2006, 21, 27.

[19] S. H. Lim, A. G. Caster, S. R. Leone, Phys. Rev. A 2005, 72, 041803.

[20] S. H. Lim, A. G. Caster, S. R. Leone, Opt. Lett. 2007, 32, 1332.

[21] T. W. Kee, M. T. Cicerone, Opt. Lett. 2004, 29, 2701.

[22] H. Kano, H. Hamaguchi, J. Raman Spectrosc. 2006, 37, 411.

[23] B. von Vacano, L. Meyer, M. Motzkus, J. Raman Spectrosc. 2007, 38, 916.

[24] L. Lepetit, G. Cheriaux, M. Joffre, J. Opt. Soc. Am. B 1995, 12, 2467.

[25] P. Bornhauser, G. Calzaferri, J. Phys. Chem. 1996, 100, 2035.

[26] H. C. Liou, J. Pretzer, Thin Solid Films 1998, 335, 186.

[27] B. C. Chen, S. H. Lim, J. Phys. Chem. B 2008, 112, 3653. 